\title{
A utilização da grounded theory em estudos sobre educação inclusiva
}

\author{
Marisa Carvalho' \\ 1Universidade Católica Portuguesa, Faculdade de Educação e Psicologia, Centro de \\ Investigação para o Desenvolvimento Humano, Portugal | mscarvalho@porto.ucp.pt | \\ https://orcid.org/0000-0002-6169-8567
}

\begin{abstract}
Resumo: Nas últimas décadas, o debate acerca da educação inclusiva tem vindo a ganhar relevo do ponto de vista da investigação educacional. A grounded theory é uma metodologia particularmente útil neste âmbito na medida em que poderá contribuir para a construção de teoria acerca das experiências de inclusão em múltiplos contextos, potenciando o caráter empoderador de modelos e teorias dirigidas para públicos em maior risco de exclusão. Neste trabalho, de natureza exploratória, pretendemos explorar de que modo a grounded theory tem vindo a ser utilizada na investigação em educação inclusiva, identificando as principais dimensões estudadas e opções metodológicas adotadas. Foi realizada uma pesquisa na base de dados Education Resources Information Center, usando as palavras-chave "Grounded theory AND Inclusive education", tendo sido identificados 12 artigos relevantes para análise. Verificou-se alguma diversidade nas dimensões identificadas, designamente relacionadas com as políticas, com a identidade profissional do professor e com a participação de crianças e jovens em diferentes contextos educativos. Do ponto de vista metodológico, os estudos identificados parecem usar a grounded theory com maior foco nos procedimentos de codificação. Conclui-se haver necessidade e vantagens de um maior aperfeiçoamento no uso da grounded theory no sentido da construção de teoria em educação inclusiva.
\end{abstract}

Palavras-chave: Grounded Theory; Educação Inclusiva; Educação.

\section{The Use of Grounded Theory in Studies on Inclusive Education}

\begin{abstract}
In the last decades, the debate about inclusive education has gained prominence in educational research. Grounded theory is a particularly useful methodology in this scope as it can contribute to theory construction about the experiences of inclusion in multiple contexts, enhancing the empowering character of models and theories aimed at audiences at greater risk of exclusion. In this exploratory work, we intend to explore how grounded theory has been used in inclusive education research, identifying the main dimensions studied and methodological options adopted. Data collection was carried out in the Education Resources Information Center database, using the keywords "Grounded theory AND Inclusive education", and 12 relevant articles were identified for analysis. There was some diversity of dimensions explored, namely related to policies, teachers' professional identity and children and youth participation in different educational contexts. From a methodological point of view, the studies identified seem to use grounded theory with a greater focus on coding procedures. We conclude that there is a need and advantages for further improvement in the use of grounded theory for building theory in inclusive education.
\end{abstract}

Keywords: Grounded Theory; Inclusive Education; Education.

\section{Introdução}

Nas últimas décadas, o debate acerca da educação inclusiva tem vindo a ganhar relevo do ponto de vista das políticas, da prática e da investigação educacional. Apesar da ideia generalizada de que as escolas e as sociedades devem pautar-se por valores de inclusão, e dos esforços no sentido de se conceberem políticas educativas, em Portugal e na Europa, alinhadas com estes princípios, as perspetivas e experiências individuais são, por vezes, contraditórias (Azorín \& Ainscow, 2020; McMaster, 2012; Moya, 2019).

A educação inclusiva tem sido assumida em diversos países como o direito de todos os alunos ao acesso a educação de elevada qualidade tendo por base princípios de equidade (Aiscow et al., 2011). Ainda assim, a definição de educação inclusiva é complexa e diversa, variando entre os autores (cf. Carvalho et al., 2019). Na literatura são sugeridas diferentes definições de educação inclusiva, que traduzem conceções e orientações distintas com implicações nas políticas educativas e nas práticas das escolas (Carvalho et al., 2019). 
Mais recentemente, adotam-se abordagens holísticas e compreensivas que consideram uma conceção abrangente de diversidade bem como as múltiplas dimensões dos contextos em termos de educação. Em especial, destacamos modelos abrangentes como o proposto por Azorín \& Ainscow (2020) que inclui 3 dimensões distintas na conceptualização da educação inclusiva: contextos; processos; recursos. Neste sentido, a educação inclusiva deixa de estar adstrita a perspetivas centradas na deficiência ou nas necessidades educativas especiais e alarga-se a modelos com enfoque comunitário que remetem para todos (crianças e jovens), ainda que, considerando também grupos em maior risco de exclusão ou marginalização (Göransson \& Nilholm, 2014).

O campo de estudo em "Educação Inclusiva" é amplo e complexo, na medida em que implica considerar múltiplos lentes e objetos de análise, diversos participantes, variados modos de recolha e de análise de dados. Implica também que a investigação seja, ela mesma, inclusiva, integrando múltiplas vozes e complexas leituras.

A grounded theory poderá ser um modelo de investigação a adotar neste âmbito, na medida em que permite explorar dados qualitativos relativos à experiência e à atribuição de significados, capazes de ilustrar dimensões pessoais, interativas e históricas da realidade pessoal (Henwood \& Pidgeon, 1995, 2003; Lee et al., 1999; Pidgeon \& Henwood, 1997). Especificamente, na linha de estudos relacionados com a Educação Inclusiva, apesar da grounded theory ser uma metodologia qualitativa pouco utilizada, parece-nos que poderá ser particularmente útil na construção de teoria acerca das experiências de inclusão em múltiplos contextos, potenciando o caráter empoderador de modelos e teorias dirigidas para públicos em maior risco de exclusão.

Esta metodologia qualitativa tem vindo progressivamente a ser utilizada pelos investigadores no âmbito das ciências sociais e humanas, nomeadamente em diferentes áreas da Educação e da Psicologia (Fernandes \& Maia, 2001; Lee et al., 1999; Pidgeon \& Henwood, 1997). Barney Glaser e Anselm Strauss, autores originais da metodologia, conceberam-na como um modelo de investigação enraizado (grounded), que combina o rigor e a sistematização com a aproximação do investigador à realidade no sentido da teorização.

A grounded theory define-se como um processo sistemático de recolha e análise de dados tendo em vista a descoberta ou construção de teoria (Bryant \& Charmaz, 2007; Charmaz, 2003, 2006; Corbin \& Strauss, 2008; Glaser \& Strauss, 1967; Strauss \& Corbin, 1990, 1998). Com efeito, a noção de teoria é central nesta metodologia. A teorização é definida como $O$ ato de construção, a partir dos dados, de uma grelha de leitura que sistematicamente integre os diferentes conceitos em afirmações de relação. A teoria não se limita à descrição dos fenómenos, mas antes implica a explicação e predição de acontecimentos (Strauss \& Corbin, 1998).

No processo específico de construção de teoria devem ser garantidas um conjunto de condições básicas, a saber: (i) o processo iterativo, baseado na interação entre a recolha e a análise de dados; (ii) a amostragem teórica, que acontece quando as categorias encontradas começam a estabilizar e os casos novos não trazem nada de novo ao investigador; (iii) a sensibilidade teórica, que informa o processo dialético constante entre os dados e os conceitos; (iv) os códigos, memorandos e conceitos necessários à análise; (v) a comparação constante; (vi) a saturação teórica; (vii) o ajustamento, funcionalidade, relevância e modificabilidade e; (viii) a teoria substantiva, uma teoria relativamente específica a um grupo e/ou lugar, que se aplica a tópicos de uma disciplina (e.g., Bryant \& Charmaz, 2007; Charmaz, 2003, 2006; Corbin \& Strauss, 2008; Glaser \& Strauss, 1967; Strauss \& Corbin, 1990, 1994, 1998). Além disso, seguem-se procedimentos específicos de codificação, onde se destacam as operações de comparação e questionamento constantes, bem como a codificação aberta, axial e seletiva (Strauss \& Corbin, 1998). 


\section{Metodologia}

Com este trabalho de caráter exploratório, pretendemos identificar de que modo a grounded theory tem vindo a ser utilizada na investigação em educação inclusiva, identificando as principais questões exploradas e opções metodológicas adotadas. Assim, pretende-se apresentar os contributos de alguns estudos de grounded theory para o estado da arte sobre Educação Inclusiva bem como discutir a respetiva utilização da metodologia.

Para a seleção dos estudos, foi realizada uma pesquisa na Education Resources Information Center (ERIC), base de dados eletrónica de revistas indexadas em Educação. Esta base de dados foi escolhida por ser uma base de dados orientada para trabalhos na área da Educação, âmbito deste estudo exploratório. Foi realizada pesquisa de textos usando as palavras-chave "grounded theory AND inclusive education" e considerado o período de 2010-2021. Considerando estes critérios, foram encontrados 12 artigos, conforme tabela 1. Exploramos (1) as questões da educação inclusiva trabalhadas em cada estudo com recurso à grounded theory bem como (2) os procedimentos metodológicos adotados nestes mesmos estudos. Com efeito, procedeu-se (1) à codificação temática considerando o tópico/problema de investigação de cada estudo e (2) à codificação categorial dos procedimentos metodológicos adotados considerando as condições de aplicação da metodológica descritos na introdução deste trabalho.

\section{Resultados}

$\mathrm{Na}$ tabela 1 apresentam-se os estudos considerados, identificando-se as palavras-chave para melhor análise do conteúdo dos mesmos, bem como a referência ao excerto onde se refere o uso da grounded theory para melhor compreensão das opções metodológicas.

A apreciação dos estudos selecionados revela um certo interesse pela utilização da metodologia para explorar aspetos específicos da educação inclusiva. Uma parte dos estudos selecionados refere-se a condições relacionadas com a participação de crianças, adolescentes e jovens adultos em contextos escolares ou de pares $(n=6)$. Destacamos, por exemplo, o trabalho de Leeuw et al (2018) relacionado com as estratégias utilizadas pelos professores para facilitar a participação em sala de aula de alunos com problemas comportamentais ou o trabalho de Strnadová et al (2015) acerca da inclusão de alunos com deficiência no ensino superior. Alguns estudos assinalam aspetos relacionados com a identidade e especificidades associadas ao ser professor em contextos que se esperam inclusivos (Suc et al, 2017; Thorius, 2016; Waitoller, 2016). Outros estudos centram-se nas políticas educativas, identificando criticamente questões relacionadas com a implementação de modelos e práticas de educação inclusiva $(n=2)$ (Engelbrecht et al, 2016; Hodkinson, 2013). Finalmente, um dos estudos centra-se no uso de metodologias de investigação participativas e no seu papel em educação inclusiva (Eldera \& Odoyob, 2018). Se por um lado, esta diversidade de problemas de investigação põe em evidência a complexidade do campo de estudo, por outro lado, aponta para a necessidade de alguma sistematização e articulação dos resultados dos diferentes estudos.

Foi, também, objetivo deste trabalho explorar as características metodológicas dos estudos de grounded theory aqui incluídos. Ainda assim, tratando-se de um estudo exploratório, optamos por uma análise global do uso da metodologia nestes estudos. A maioria dos estudos aqui incluídos refere-se à grounded theory na secção de análise de dados, assinalando essencialmente aspetos relacionados com o processo de codificação para explicitar o uso desta metodologia (e.g. Sempowicz et al., 2018; Waitoller, 2016). Em alguns casos, há referência ao modelo de grounded theory adotado (e.g. Eldera \& Odoyob, 2018). Outras características da grounded theory, como a amostragem teórica, a saturação teórica ou a teoria substantiva, não são referenciadas nos estudos aqui incluídos. Há, ainda, a referir a existência de dois estudos que não referem explicitamente a grounded theory como metodologia, mas adotam a referência "comparação constante" como equivalente, pelo que foram aqui considerados (e.g. Engelbrecht et al., 2016). 
Parece, então, que os estudos aqui apresentados consideram alguns elementos que constituem as condições básicas da grounded theory, mas não a sua totalidade. Reconhecem-se algumas dificuldades na operacionalização da amostragem e saturação teóricas bem como a ausência de referências aos critérios e formas de avaliação da teoria e do processo de investigação qualitativa.

Tabela 1. Estudos de grounded theory em educação inclusiva

\begin{tabular}{|c|c|c|c|}
\hline $\begin{array}{c}\text { Autores } \\
\text { (Revista, Ano) }\end{array}$ & Título & Palavras-chave & Uso da grounded theory \\
\hline $\begin{array}{l}\text { Eldera \& } \\
\text { Odoyob, } 2018 \\
\text { (International } \\
\text { Journal of } \\
\text { Qualitative } \\
\text { Studies in } \\
\text { Education) }\end{array}$ & $\begin{array}{l}\text { Multiple } \\
\text { methodologies: } \\
\text { using community- } \\
\text { based participatory } \\
\text { research and } \\
\text { decolonizing } \\
\text { methodologies in } \\
\text { Kenya }\end{array}$ & $\begin{array}{l}\text { Community-based } \\
\text { participatory } \\
\text { research (CBPR); } \\
\text { decolonizing } \\
\text { methodologies; } \\
\text { qualitative } \\
\text { research; Kenya; } \\
\text { inclusive education }\end{array}$ & $\begin{array}{l}\text { "In this project, we used a qualitative } \\
\text { approach to data analysis (i.e. } \\
\text { grounded theory) informed by CBPR } \\
\text { and decolonizing methods" (p. 309) }\end{array}$ \\
\hline $\begin{array}{l}\text { Engelbrecht et } \\
\text { al, } 2016 \\
\text { (International } \\
\text { Journal of } \\
\text { Inclusive } \\
\text { Education) }\end{array}$ & $\begin{array}{l}\text { The idealism of } \\
\text { education policies } \\
\text { and the realities in } \\
\text { schools: the } \\
\text { implementation of } \\
\text { inclusive education } \\
\text { in South Africa }\end{array}$ & $\begin{array}{l}\text { Education policy; } \\
\text { policy } \\
\text { implementation; } \\
\text { inclusive } \\
\text { education; South } \\
\text { African context; } \\
\text { inclusive practice }\end{array}$ & $\begin{array}{l}\text { "Systematically working through the } \\
\text { stages of the constant comparative } \\
\text { analysis method" (p.528) }\end{array}$ \\
\hline $\begin{array}{l}\text { Hodkinson, } \\
2013 \text { (Journal } \\
\text { for Critical } \\
\text { Education } \\
\text { Policy Studies) }\end{array}$ & $\begin{array}{l}\text { Inclusion 'All } \\
\text { present and } \\
\text { correct?' A critical } \\
\text { analysis of New } \\
\text { Labour's inclusive } \\
\text { education policy in } \\
\text { England }\end{array}$ & $\begin{array}{l}\text { Foreign Countries, } \\
\text { Educational Policy, } \\
\text { Inclusion, } \\
\text { Epistemology, } \\
\text { Equal Education, } \\
\text { Policy Formation, } \\
\text { Educational } \\
\text { History, Politics of } \\
\text { Education, } \\
\text { Government Role, } \\
\text { Semi Structured } \\
\text { Interviews, } \\
\text { Teacher Attitudes, } \\
\text { Mainstreaming, } \\
\text { Grounded Theory }\end{array}$ & $\begin{array}{l}\text { "The interview data were analysed } \\
\text { by the employment of grounded } \\
\text { theory which focused on the } \\
\text { discovery of theory from data } \\
\text { (Glaser and Strauss, 1967)" (p. 245) }\end{array}$ \\
\hline $\begin{array}{l}\text { Isaksson et al, } \\
2010 \\
\text { (International } \\
\text { Journal of } \\
\text { Inclusive } \\
\text { Education) }\end{array}$ & $\begin{array}{l}\text { 'Pupils with special } \\
\text { educational } \\
\text { needs': a study of } \\
\text { the assessments } \\
\text { and categorising } \\
\text { processes } \\
\text { regarding pupils' } \\
\text { school difficulties } \\
\text { in Sweden }\end{array}$ & $\begin{array}{l}\text { Assessment; } \\
\text { special education } \\
\text { needs; inclusive } \\
\text { education; special } \\
\text { education }\end{array}$ & $\begin{array}{l}\text { "We choose to use the grounded } \\
\text { theory approach to analyse the } \\
\text { interview data. (p.138) }\end{array}$ \\
\hline $\begin{array}{l}\text { Leeuw et al, } \\
2018\end{array}$ & $\begin{array}{l}\text { Teacher strategies } \\
\text { to support the } \\
\text { social participation } \\
\text { of students with } \\
\text { SEBD in the } \\
\text { regular classroom }\end{array}$ & $\begin{array}{l}\text { Internalising and } \\
\text { externalising } \\
\text { behavioural } \\
\text { difficulties; primary } \\
\text { schoolteachers; } \\
\text { inclusive education }\end{array}$ & $\begin{array}{l}\text { "For the analyses of the qualitative } \\
\text { data a multi-grounded theory } \\
\text { approach was used (Goldkuhl and } \\
\text { Cronholm 2010)" (p. 415) }\end{array}$ \\
\hline $\begin{array}{l}\text { Loreman et al, } \\
2016 \text { (School } \\
\text { Effectiveness } \\
\text { and School } \\
\text { Improvement) }\end{array}$ & $\begin{array}{l}\text { A Canada-Ukraine } \\
\text { collaborative } \\
\text { initiative for } \\
\text { introducing } \\
\text { inclusive education } \\
\text { for children with } \\
\text { disabilities in } \\
\text { Ukraine: } \\
\text { participant } \\
\text { perspectives }\end{array}$ & $\begin{array}{l}\text { Inclusive } \\
\text { education; } \\
\text { Ukraine; } \\
\text { participant } \\
\text { research }\end{array}$ & $\begin{array}{l}\text { "This study is situated within the } \\
\text { paradigm of grounded theory } \\
\text { research." (pp. 30-31) }\end{array}$ \\
\hline
\end{tabular}




\begin{tabular}{|c|c|c|c|}
\hline $\begin{array}{c}\text { Autores } \\
\text { (Revista, Ano) }\end{array}$ & Título & Palavras-chave & Uso da grounded theory \\
\hline $\begin{array}{l}\text { Østvik et al, } \\
2018 \\
\text { (European } \\
\text { Journal of } \\
\text { Special Needs } \\
\text { Education) }\end{array}$ & $\begin{array}{l}\text { 'So, how does one } \\
\text { define a } \\
\text { friendship?': } \\
\text { Identifying } \\
\text { friendship among } \\
\text { students using } \\
\text { AAC in inclusive } \\
\text { education settings }\end{array}$ & $\begin{array}{l}\text { Friendship; } \\
\text { children; inclusive } \\
\text { education; } \\
\text { augmentative and } \\
\text { alternative } \\
\text { communication } \\
\text { (AAC) }\end{array}$ & $\begin{array}{l}\text { "The transcriptions were analysed } \\
\text { using a constructivist grounded } \\
\text { theory approach (Charmaz 2014)." } \\
\text { (p. 338) }\end{array}$ \\
\hline $\begin{array}{l}\text { Sempowicz et } \\
\text { al, } 2018 \\
\text { (International } \\
\text { Journal of } \\
\text { Inclusive } \\
\text { Education) }\end{array}$ & $\begin{array}{l}\text { Identifying } \\
\text { obstacles and } \\
\text { opportunities for } \\
\text { inclusion in the } \\
\text { school curriculum } \\
\text { for children } \\
\text { adopted from } \\
\text { overseas: } \\
\text { developmental and } \\
\text { social } \\
\text { constructionist } \\
\text { perspectives }\end{array}$ & $\begin{array}{l}\text { Attachment } \\
\text { disruption; } \\
\text { childhood } \\
\text { development; } \\
\text { complex trauma; } \\
\text { inclusive } \\
\text { education; } \\
\text { intercountry } \\
\text { adoption; social } \\
\text { construction }\end{array}$ & $\begin{array}{l}\text { "The constant comparative approach } \\
\text { enabled themes and subthemes to } \\
\text { evolve" (p. 611) }\end{array}$ \\
\hline $\begin{array}{l}\text { Strnadová et } \\
\text { al, } 2015 \\
\text { (International } \\
\text { Journal of } \\
\text { Inclusive } \\
\text { Education) }\end{array}$ & $\begin{array}{l}\text { Voices of } \\
\text { university students } \\
\text { with disabilities: } \\
\text { inclusive education } \\
\text { on the tertiary level } \\
\text { - a reality or a } \\
\text { distant dream? }\end{array}$ & $\begin{array}{l}\text { Higher education } \\
\text { students with } \\
\text { disabilities; } \\
\text { inclusive } \\
\text { education; barriers } \\
\text { types of support } \\
\text { coping strategies }\end{array}$ & $\begin{array}{l}\text { "The semi-structured interviews were } \\
\text { transcribed verbatim and analysed } \\
\text { using the grounded theory approach } \\
\text { (Strauss and Corbin, 1999)." } \\
\text { (p.1084) }\end{array}$ \\
\hline $\begin{array}{l}\text { Suc et al, } 2017 \\
\text { (International } \\
\text { Journal of } \\
\text { Inclusive } \\
\text { Education) }\end{array}$ & $\begin{array}{l}\text { The role of inter- } \\
\text { professional } \\
\text { collaboration in } \\
\text { developing } \\
\text { inclusive } \\
\text { education: } \\
\text { experiences of } \\
\text { teachers and } \\
\text { occupational } \\
\text { therapists in } \\
\text { Slovenia }\end{array}$ & $\begin{array}{l}\text { Inter-professional } \\
\text { collaboration; } \\
\text { inclusive } \\
\text { education; children } \\
\text { with special needs; } \\
\text { occupational } \\
\text { therapy; primary } \\
\text { school teachers }\end{array}$ & $\begin{array}{l}\text { "Qualitative content analysis } \\
\text { (Strauss and Corbin 1998) was used } \\
\text { to analyse focus group interviews } \\
\text { and individual interviews" (p.946) }\end{array}$ \\
\hline $\begin{array}{l}\text { Thorius, } 2016 \\
\text { (International } \\
\text { Journal of } \\
\text { Inclusive } \\
\text { Education) }\end{array}$ & $\begin{array}{l}\text { Stimulating } \\
\text { tensions in special } \\
\text { education } \\
\text { teachers' figured } \\
\text { world: an approach } \\
\text { toward inclusive } \\
\text { education }\end{array}$ & $\begin{array}{l}\text { Special education } \\
\text { teacher identity; } \\
\text { teacher learning; } \\
\text { inclusive } \\
\text { education; figured } \\
\text { worlds; technical } \\
\text { assistance }\end{array}$ & $\begin{array}{l}\text { "I employed constructivist grounded } \\
\text { theory as discussed by Charmaz } \\
\text { (2006)" (p.1332) }\end{array}$ \\
\hline $\begin{array}{l}\text { Waitoller, } 2016 \\
\text { (School } \\
\text { Effectiveness } \\
\text { and School } \\
\text { Improvement) }\end{array}$ & $\begin{array}{l}\text { Professional } \\
\text { inquiry for inclusive } \\
\text { education: learning } \\
\text { amidst institutional } \\
\text { and professional } \\
\text { boundaries }\end{array}$ & $\begin{array}{l}\text { Inclusive } \\
\text { education; inquiry; } \\
\text { professional } \\
\text { learning }\end{array}$ & $\begin{array}{l}\text { "We used analytical tools from } \\
\text { Grounded Theory to analyze videos } \\
\text { of meetings between university and } \\
\text { school personnel, field notes, and } \\
\text { meeting artifacts." (p. 62) }\end{array}$ \\
\hline
\end{tabular}

\section{Conclusões}

A grounded theory é uma metodologia de investigação qualitativa que progressivamente tem vindo a ganhar adeptos, nomeadamente na Educação (Charmaz, 2003, 2006; Pidgeon \& Henwood, 1997). O seu objetivo último é gerar teoria e, deste modo, contribuir para leituras integradas acerca dos fenómenos em estudo. Por este motivo, parece-nos ser uma metodologia capaz de potenciar a investigação sobre a Educação Inclusiva. 
Com este trabalho, procuramos explorar de que modo a grounded theory tem sido utilizada em estudos sobre educação inclusiva e os contributos destes mesmos estudos para o campo em análise. Sendo um estudo exploratório, justifica desenvolvimentos futuros com recurso a estudos mais aprofundados de revisão de literatura.

Os estudos realizados na Educação Inclusiva, e aqui apresentados, descrevem pontos essenciais de aplicação da metodologia. Ainda assim, mantém-se a questão da garantia das condições básicas propostas pelos autores originais (e.g. procedimentos específicos de codificação ou de amostragem teórica). A análise exploratória dos estudos aqui incluídos traduz a necessidade de um maior aperfeiçoamento no uso da metodologia no sentido da construção de teoria em educação inclusiva, por considerarmos a relevância da mesma na exploração de dimensões específicas que se prendem com as experiências subjetivas das pessoas nos múltiplos contextos de vida.

\section{Referências}

Ainscow, M., Dyson, A., Goldrick, S., \& West, M. (2011). Developing equitable education systems. Routledge.

Azorín, C. \& Ainscow, M. (2020). Guiding schools on their journey towards inclusion, International Journal of Inclusive Education, 24(1), 58-76, DOI: 10.1080/13603116.2018.1450900

Bryant, A. \& Charmaz, K. (Eds.). (2007). The sage handbook of grounded theory. Sage Publications.

Carvalho, M. (2019, 30 janeiro). Educação inclusiva. Das políticas às práticas (comunicação). CFAEMC.

Charmaz, K. (2003). Grounded theory. In J. A. Smith (Ed.), Qualitative psychology: a practical guide to research methods (1st ed., pp.81-110). Sage Publications.

Charmaz, K. (2006). Constructing grounded theory: a practical guide through qualitative analysis. Sage Publications.

Corbin, J. M. \& Strauss, A. L. (2008). Basics of qualitative research: techniques and procedures for developing grounded theory. Sage Publications.

Elder, B. \& Odoyo, K. (2018) Multiple methodologies: using community-based participatory research and decolonizing methodologies in Kenya, International Journal of Qualitative Studies in Education, 31(4), 293-311, DOI: 10.1080/09518398.2017.1422290

Engelbrecht, P., Nel, M., Smit, S. \& Deventer, M. (2015). The idealism of education policies and the realities in schools: the implementationof inclusive education in South Africa, International Journal of Inclusive Education, 20(5), 520-535, DOI: 10.1080/13603116.2015.1095250

Fernandes, E. \& Maia, A. (2001). Grounded theory. In E. M. Fernandes \& L. S. Almeida (Eds.), Métodos e técnicas de avaliação. Contributos para a prática e investigação psicológicas (pp.49-76). CEEP/UM.

Glaser, B. G. \& Strauss, A. L. (1967). The discovery of grounded theory: Strategies for qualitative research. Aldine de Gruyter.

Göransson, K. \& Nilholm, C. (2014) Conceptual diversities and empirical shortcomings - a critical analysis of research on inclusive education, European Journal of Special Needs Education, 29(3), 265-280, DOI: 10.1080/08856257.2014.933545

Henwood, K. \& Pidgeon, N. (1995). Grounded theory and psychological research. The Psychologist, 8(3), 115-118.

Henwood, K. \& Pidgeon, N. (2003). Grounded theory in psychological research. In P. M. Camic, J. E. Rhodes \& L. Yardley (Eds.), Qualitative research in psychology: expanding perspectives in methodology and design (1st. ed., 131-155). American Psychological Association.

Hodkinson, A. (2013). Inclusion "all present and correct?" A critical analysis of new labour's inclusive education policy in England. Journal for Critical Education Policy Studies, 11(4), 242-262. 
Isaksson, J., Lindqvist, R. \& Bergström, E. (2010). 'Pupils with special educational needs': a study of the assessments and categorising processes regarding pupils' school difficulties in Sweden, International Journal of Inclusive Education, 14(2), 133-151, DOI: $10.1080 / 13603110802504176$

Lee, T. W., Mitchell, T. R. \& Sablynski, C. J. (1999). Qualitative research in organizational and vocational psychology, 1979-1999. Journal of Vocational Behavior, 55, 161-187.

Leeuw, R., de Boer, A., Bijstra, J. \& Minnaert, A. (2018) Teacher strategies to support the social participation of students with SEBD in the regular classroom, European Journal of Special Needs Education, 33(3), 412-426, DOI: 10.1080/08856257.2017.1334433

Loreman, T., McGhie-Richmond, D., Kolupayeva, A., Taranchenko, O., Mazin, D., Crocker, C. \& Petryshyn, R. (2016). A Canada-Ukraine collaborative initiative for introducing inclusive education for children with disabilities in Ukraine: participant perspectives, School Effectiveness and School Improvement, 27(1), 24-44, DOI: 10.1080/09243453.2015.1018912

McMaster, C. (2012). Ingredients for inclusion: lessons from the literature. KAIRARANGA 13(2), 1122.

Moya, E. (2019). Hacia una educación inclusiva para todos. Nuevas contribuciones. Profesorado. Revista de Currículum y Formación de Profesorado, 23(1), 1-9. DOI:10.30827/profesorado.v23i1.9142

Østvik, J., Ytterhus, B. \& Balandin, S. (2018). 'So, how does one define a friendship?': Identifying friendship among students using AAC in inclusive education settings, European Journal of Special Needs Education, 33(3), 334-348, DOI: 10.1080/08856257.2017.1312799

Pidgeon, N. \& Henwood, K. (1997). Using grounded theory in psychological research. In N. Hayes (Ed.), Doing qualitative analysis in psychology (1st., pp.245-275). Psychology Press.

Sempowicz, T., Howard, J., Tambyah, M. \& Carrington, S. (2018) Identifying obstacles and opportunities for inclusion in the school curriculum for children adopted from overseas: developmental and social constructionist perspectives, International Journal of Inclusive Education, 22(6), 606-621, DOI: 10.1080/13603116.2017.1390004

Strauss, A. \& Corbin, J. (1990). Basics of qualitative research: Grounded theory procedures and techniques (1st. ed.). Sage.

Strauss, A. \& Corbin, J. (1994). Grounded theory methodology. An overiew. In N. Denzin \& Y. Lincoln (Eds.). Handbook of qualitative research (1st. ed., pp. 273-285). Sage Publications.

Strauss, A. \& Corbin, J. (1998). Basics of qualitative research. Techniques and procedures for developing grounded theory (2nd ed.). Sage Publications.

Strnadová, I., Hájková, V. \& Květoňová, L. (2015) Voices of university students with disabilities: inclusive education on the tertiary level - a reality or a distant dream?, International Journal of Inclusive Education, 19(10), 1080-1095, DOI: 10.1080/13603116.2015.1037868

Suc, L., Bukovec, B. \& Karpljuk, D. (2017) The role of inter-professional collaboration in developing inclusive education: experiences of teachers and occupational therapists in Slovenia, International Journal of Inclusive Education, 21(9), 938-955, DOI: $10.1080 / 13603116.2017 .1325073$

Thorius, K. (2016) Stimulating tensions in special education teachers' figured world: an approach toward inclusive education, International Journal of Inclusive Education, 20(12), 1326-1343, DOI: $10.1080 / 13603116.2016 .1168877$

Waitoller, F., Kozleski, E. \& Gonzalez, T. (2016) Professional inquiry for inclusive education: learning amidst institutional and professional boundaries, School Effectiveness and School Improvement, 27(1), 62-79, DOI: 10.1080/09243453.2014.908929 